\title{
Effect of electrolytic hydrogen saturation on deformation mechanisms of Fe-17Cr-13Ni-3Mo-0.01C austenitic stainless steel during cold rolling
}

\author{
E. V. Melnikov ${ }^{\dagger}$ M. Yu. Panchenko, K. A. Reunova, E. G. Astafurova \\ ${ }^{\dagger}$ melnickow-jenya@yandex.ru
}

Institute of Strength Physics and Materials Science SB RAS, Tomsk, 634055, Russia

\begin{abstract}
In this work, we investigated the effect of the current density during electrolytic hydrogen-charging before deformation on the mechanisms of plastic deformation of stable austenitic stainless steel Fe-17Cr-13Ni-3Mo-0.01C during rolling. Using transmission electron microscopy and backscattered electron diffraction, it has been shown that plastic deformation by rolling at room temperature with $25 \%$ reduction causes the formation of a high density of crystal lattice defects in the initially coarse-grained steel samples. The main deformation mechanism during rolling is dislocation slip, which is accompanied by mechanical twinning as an additional mechanism contributing the structure fragmentation. Regardless of the mode of preliminary hydrogen-charging, it promotes more active development of twinning. The higher the current density during hydrogen pre-charging, the higher the density of twin boundaries and dislocation density in the steel structure during rolling. After hydrogen-charging at a current density of $200 \mathrm{~mA} / \mathrm{cm}^{2}$, the $\gamma \rightarrow \varepsilon$ transformation is also realized upon deformation. At the same time, an increase in the current density upon hydrogen pre-charging from 10 to $200 \mathrm{~mA} / \mathrm{cm}^{2}$ does not contribute to a significant increase in the concentration of hydrogen adsorbed by the material ( $0.0017-0.0018$ mass.\%), which, together with microstructural studies, indicates a different distribution of hydrogen in the samples immediately before deformation and the important role of the hydrogen concentration gradient on the deformation mechanisms of the steel during subsequent rolling. With a decrease in the deformation temperature (due to the cooling of the samples before rolling to the temperature of liquid nitrogen), hydrogen-charging also causes an increase in the linear density of twin boundaries and the formation of $\varepsilon$-martensite, but these effects are more significant compared to room-temperature deformation. The data obtained indicate that at similar values of the concentration of adsorbed hydrogen in the structure of steels, the saturation mode affects the deformation mechanism and phase transformations in austenitic steel.
\end{abstract}

Keywords: austenitic steel, plastic deformation, hydrogen-charging, microstructure.

УДК: $621.77 ; 538.91$

\section{Влияние электролитического насыщения водородом} на механизмы деформации аустенитной нержавеющей стали
01X17Н13М3 при холодной прокатке

\author{
Мельников Е. В. ${ }^{\dagger}$, Панченко М. Ю., Реунова К. А., Астафурова Е. Г. \\ Институт физики прочности и материаловедения СО РАН, Томск, 634055, Россия
}

В работе исследовали влияние плотности тока при электролитическом насыщении водородом, предшествующем деформированию, на механизмы пластической деформации стабильной аустенитной нержавеющей стали 01X17Н13М3 при прокатке. С использованием методов просвечивающей электронной микроскопии и дифракции обратнорассеянных электронов показано, что пластическая деформация прокаткой при комнатной температуре со степенью осадки 25\% вызывает формирование высокой плотности дефектов кристаллического строения в исходно крупнокристаллических стальных образцах. Основным механизмом деформации при прокатке выступает дислокационное скольжение, которое сопровождается развитием механического двойникования как дополнительного механизма, способствующего фрагментации структуры. Независимо от режима предварительного насыщения стальных образцов водородом, оно способствует более активному развитию двойникования. Повышение плотности 
тока при насыщении вызывает увеличение плотности двойниковых границ и плотности дислокаций в структуре прокатанных образцов. После наводороживания при плотности тока $200 \mathrm{MA} / \mathrm{cm}^{2}$, при деформации также реализуется $\gamma \rightarrow \varepsilon$ превращение. При этом увеличение плотности тока при предварительном насыщении образцов водородом от 10 до $200 \mathrm{~mA} / \mathrm{cm}^{2}$ не способствует значительному увеличению концентрации адсорбированного материалом водорода (0.0017-0.0018 масс.\%), что в совокупности с микроструктурными исследованиями свидетельствует о различном распределении водорода в образцах непосредственно перед деформированием и о важной роли градиента концентрации водорода у поверхности на механизмы деформации стали при последующей прокатке. При понижении температуры деформации (за счет охлаждения образцов перед прокаткой до температуры жидкого

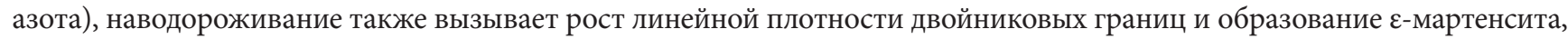
но эти эффекты более значительны по сравнению с комнатной температурой деформации. Полученные данные свидетельствуют о том, что при близких значениях концентрации адсорбированного водорода в структуре сталей, режим насыщения влияет на механизм деформации и деформационные фазовые превращения в аустенитной стали.

Ключевые слова: аустенитная сталь, пластическая деформация, наводороживание, микроструктура.

\section{1. Введение}

Аустенитные нержавеющие стали (АНС) благодаря хорошей пластичности, свариваемости, стойкости к коррозии и окислению в широком диапазоне температур широко используются в промышленности [1-3]. Под влиянием низких температур и/или деформации АНС могут испытывать $\gamma \rightarrow \varepsilon$ и $\gamma \rightarrow \alpha^{\prime}$ мартенситные превращения [4-9]. Количество деформационных

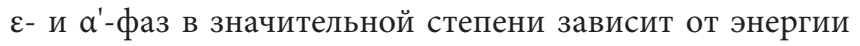
дефекта упаковки (ЭДУ) стали $[4,5]$. Различают метастабильные (с низкой ЭДУ и значительным количеством мартенситных фаз) $[6,8]$ и стабильные материалы, в которых превращение почти полностью подавлено (с высокой ЭДУ) [7,9]. Величина ЭДУ также контролирует механизмы пластической деформации сталей при высоких значениях облегчено поперечное скольжение дислокаций и затруднено развитие механического двойникования [10].

Сталь 01Х17Н13М3 при холодной пластической деформации устойчива к фазовым (мартенситным) превращениям, ее ЭДУ при комнатной температуре составляет 34 мДж/м² [11]. Отсутствие склонности к образованию деформационного мартенсита способствует высокой устойчивости стали 01Х17Н13М3 к водородному охрупчиванию [12,13]. При этом ряд экспериментальных работ свидетельствует о том, что само по себе насыщение водородом снижает ЭДУ аустенитных сталей и способствует развитию двойникования и фазовых переходов [14,15]. Как правило, влияние электролитического (поверхностного) наводороживания на механизмы деформации сталей изучают в экспериментах по статическому растяжению $[16,17]$. При использовании иных схем нагружения (прокатка, кручение под высоким давлением, прессование) закономерности влияния водорода на механизмы деформации сталей изучены меньше $[18,19]$, при этом водородно-индуцируемые эффекты могут быть иными в силу того, что высокие приложенные давления стимулируют диффузию водорода под напряжением.

Целью данной работы было изучить влияние плотности тока при электролитическом наводороживании на механизмы деформации и микроструктуру стали 01Х17Н13М3 при холодной прокатке.

\section{2. Материал и методы исследования}

Для исследования была выбрана аустенитная нержавеющая сталь 01Х17Н13M3 промышленной выплавки. Стальные пластины после химической чистки в растворе «царской водки» нагревали до температуры $1100^{\circ} \mathrm{C}$, выдерживали в течение часа и закаливали в воду комнатной температуры. Термическую обработку проводили в среде инертного газа (гелия). Для наводороживания и прокатки использовали образцы в форме пластин с размерами $10 \times 20 \times 1$ мм$^{3}$, перед обработкой их механически шлифовали и электролитически полировали в растворе $25 \Gamma \mathrm{CrO}_{3}+200$ мл $\mathrm{H}_{3} \mathrm{PO}_{4}$. Электролитическое насыщение образцов водородом проводили в $1 \mathrm{~N}$ растворе серной кислоты $\left(\mathrm{H}_{2} \mathrm{SO}_{4}\right)$ с добавлением тиомочевины $\left(\mathrm{CH}_{4} \mathrm{~N}_{2} \mathrm{~S}\right)$ в течение 5 часов при комнатной температуре. Плотность тока при насыщении водородом составляла $j=10,100,200 \mathrm{MA} / \mathrm{cm}^{2}$. Концентрацию атомов водорода определяли с использованием спектрометра LECO ONH. Прокатку наводороженных пластин осуществляли по двух режимам: I - при комнатной температуре, II - с охлаждением пластин до температуры жидкого азота перед каждым циклом прокатки. Общая степень деформации составляла $25 \%$.

Зеренную структуру исходных и деформированных образцов изучали методом анализа картин дифракции обратно рассеянных электронов (ДОЭ) с использованием сканирующего электронного микроскопа Quanta 600 FEG при ускоряющем напряжении 30 кВ. Микроструктуру изучали с помощью просвечивающего электронного микроскопа JEOL JEM-2100 (ПЭМ). Плотность дислокаций и двойниковых границ определяли по методике, описанной в [16]. Механические свойства деформированных образцов определяли в тестах на одноосное статическое растяжение при комнатной температуре и начальной скорости деформации $4 \times 10^{-4} \mathrm{c}^{-1}$ с использованием установки Instron 3369.

\section{3. Результаты и их обсуждение}

Исходные образцы АНС обладали аустенитной структурой со средним размером зерна $22.5 \pm 14.8$ мкм (наиболее вероятный размер зерна -45 мкм). Доля боль- 
шеугловых границ (БУГ) составляла $\approx 98 \%$, из них $38 \%$ - это границы $\sum 3$ двойников закалки. Плотность границ $\Sigma 3$, которую оценивали по ДОЭ-картам, была низкой $\rho_{\text {дв }}=0.06 \times 10^{6} \mathrm{M}^{-1}$. Исходные образцы обладали механическими свойствами, типичными для хромоникелевых сталей близкого состава: $\sigma_{0.2}=375 \mathrm{MПа,} \delta=65 \%$.
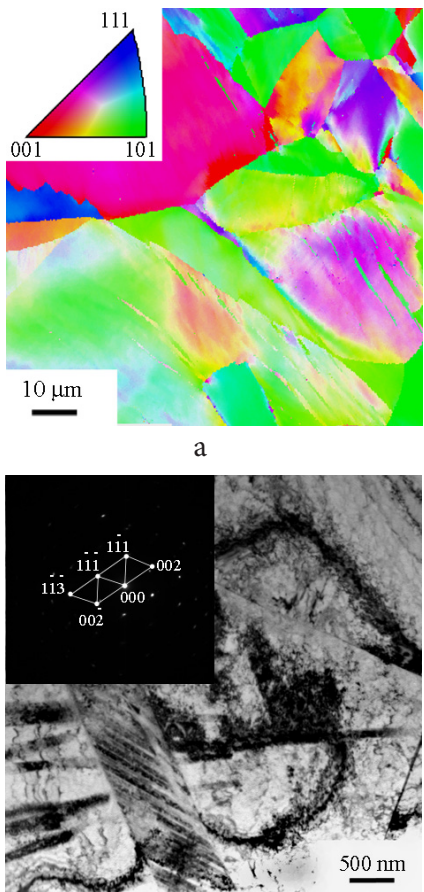

e

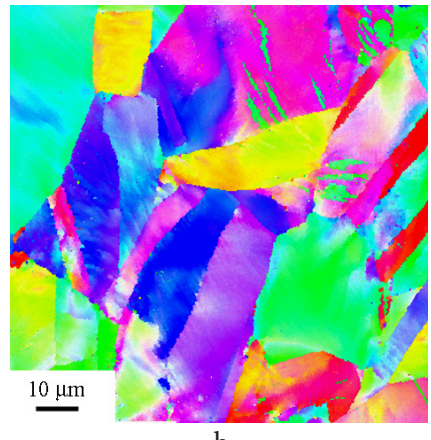

b

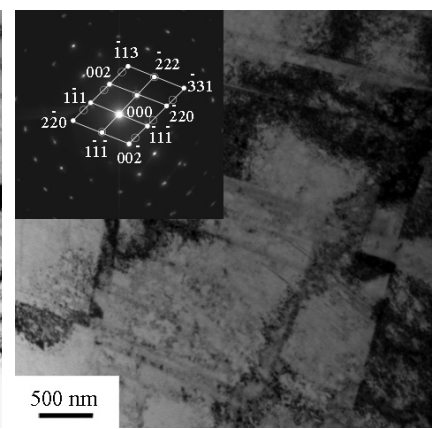

$\mathrm{f}$
Прокатка при комнатной температуре, как с охлаждением образцов, так и без него, способствует накоплению дислокаций в зернах, их фрагментации за счет образования двойниковых границ, но формирования субзеренных границ не происходит (Рис. 1a,e и $2 \mathrm{a}, \mathrm{e}$ ). На ДОЭ-картах (Рис. 1а и 2 a) изменение цветового кода

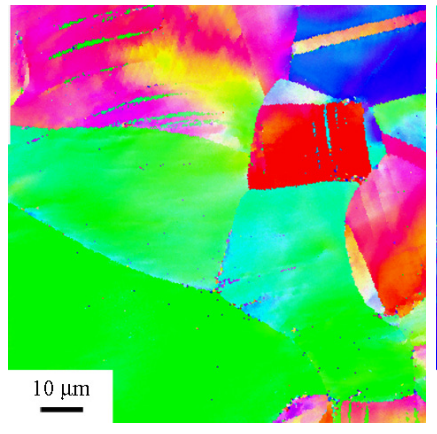

c

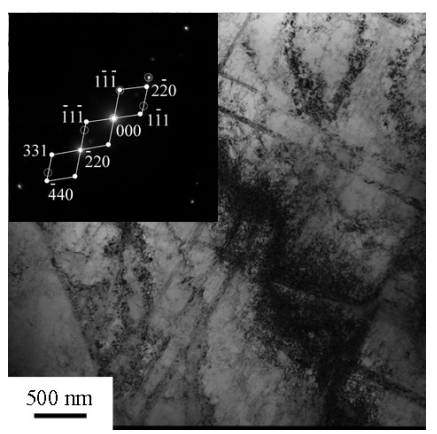

g

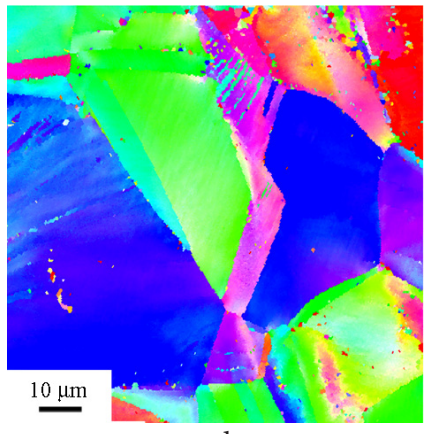

d

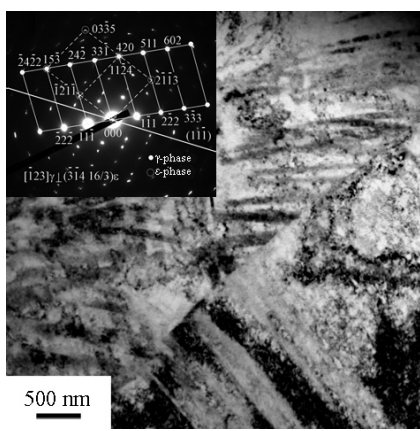

$\mathrm{h}$

Pис. 1. (Color online) Данные ДОЭ-анализа (a-d) и ПЭМ-изображения (e-h) микроструктуры стали после обработки по режиму I:

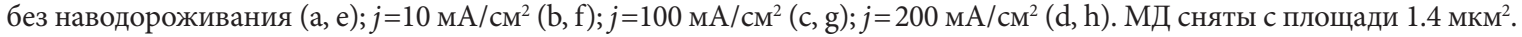

Fig. 1. (Color online) EBSD-analysis data $(\mathrm{a}-\mathrm{d})$ and TEM images $(\mathrm{e}-\mathrm{h})$ of the steel microstructure after processing in mode I: of hydrogenfree $(\mathrm{a}, \mathrm{e}) ; j=10 \mathrm{~mA} / \mathrm{cm}^{2}(\mathrm{~b}, \mathrm{f}) ; j=100 \mathrm{~mA} / \mathrm{cm}^{2}(\mathrm{c}, \mathrm{g}) ; j=200 \mathrm{~mA} / \mathrm{cm}^{2}(\mathrm{~d}, \mathrm{~h})$. Microdiffraction patterns are obtained from an area of $1.4 \mu \mathrm{m}^{2}$.

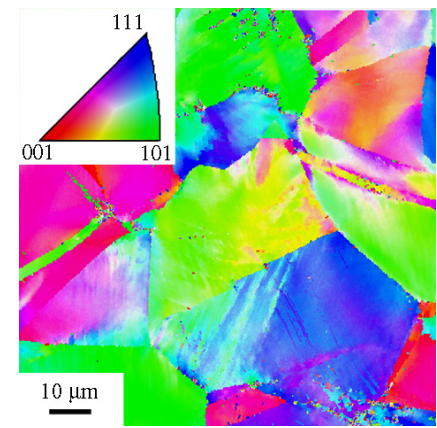

a

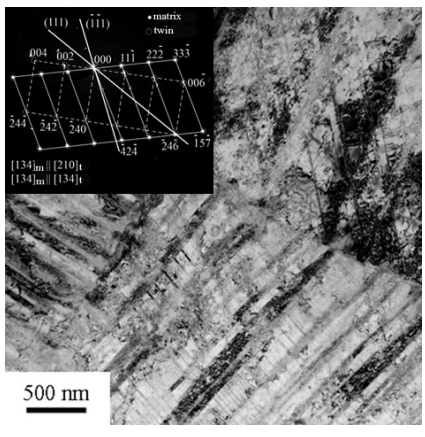

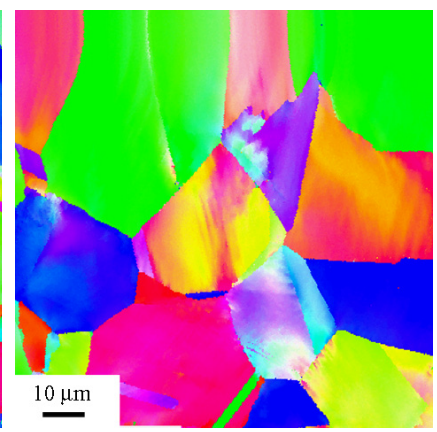

b

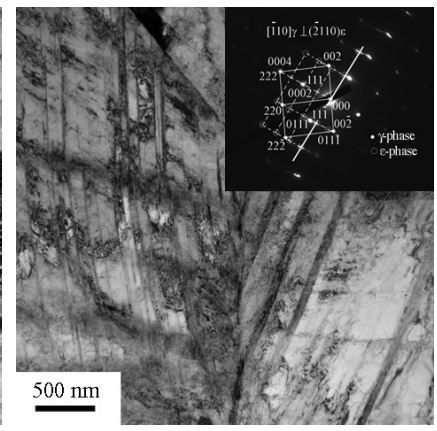

f
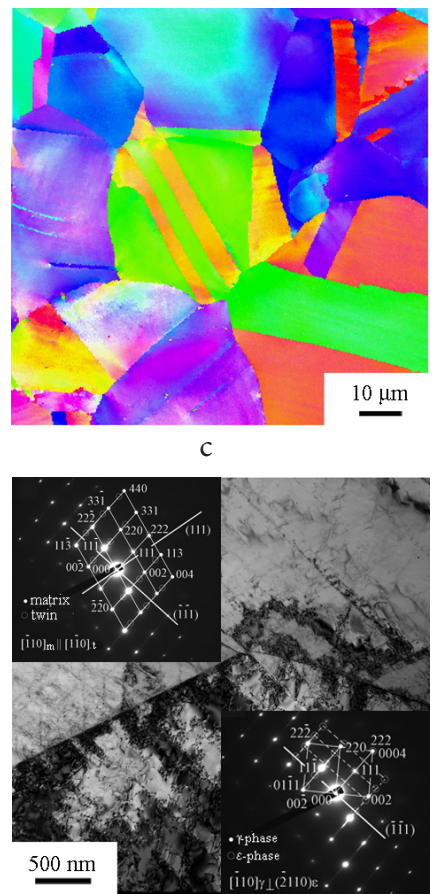

g

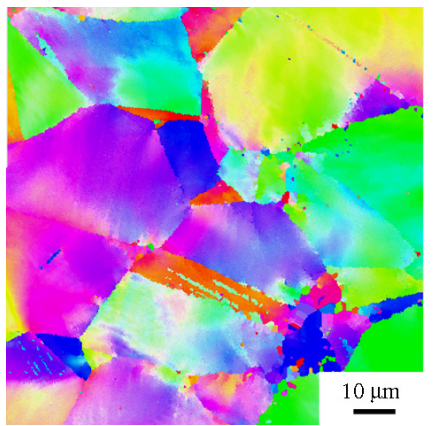

d

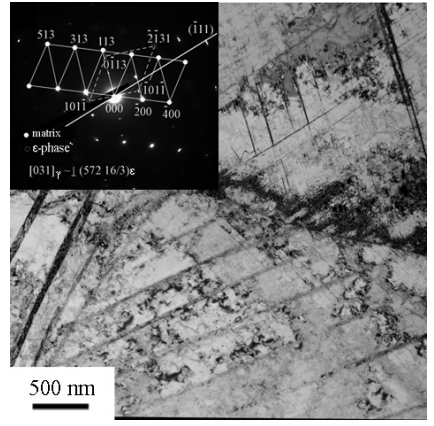

$\mathrm{h}$

Pис. 2. (Color online) Данные ДОЭ-анализа (a-d) и ПЭМ-изображения (e-h) микроструктуры стали после обработки по режиму II:

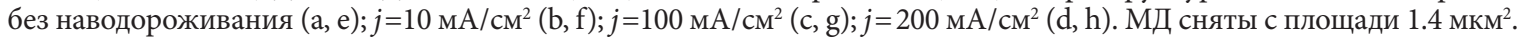

Fig. 2. (Color online) EBSD-analysis data $(\mathrm{a}-\mathrm{d})$ and TEM images $(\mathrm{e}-\mathrm{h})$ of the steel microstructure after processing in mode II: of hydrogenfree $(\mathrm{a}, \mathrm{e}) ; j=10 \mathrm{~mA} / \mathrm{cm}^{2}(\mathrm{~b}, \mathrm{f}) ; j=100 \mathrm{~mA} / \mathrm{cm}^{2}(\mathrm{c}, \mathrm{g}) ; j=200 \mathrm{~mA} / \mathrm{cm}^{2}(\mathrm{~d}, \mathrm{~h})$. Microdiffraction patterns are obtained from an area of $1.4 \mu \mathrm{m}^{2}$. 
в пределах одного зерна свидетельствует о формировании непрерывных разориентировок, не превышающих $15^{\circ}$. Помимо этого присутствуют элементы структуры с дискретными разориентировками - ламели с толщиной до 0.5 мкм, ограниченные границами 23 . Сопоставление данных ДОЭ и ПЭМ указывает на то, что это двойниковые пакеты, состоящие из тонких двойниковых ламелей, толщины которых изменяются в диапазоне 20-100 нм. Из-за ограничения разрешающей способности метода ДОЭ индивидуальные двойниковые ламели в таких пакетах и в остальном объеме зерен не разрешаются.

Метод ПЭМ дает более корректные данные о распределении деформационных двойников в исследуемых образцах (Рис. 1е и $2 \mathrm{e}$ ). После прокатки при комнатной температуре линейная плотность двойниковых границ в среднем составляет $\rho_{\text {дв }} \approx 2 \times 10^{6} \mathrm{M}^{-1}$, но в отдельныхзернах, благоприятно ориентированных для двойникования, она существенно выше (Рис. 1е, Табл. 1). Двойники реже объединены в пакеты, что затрудняет их идентификацию методом ДОЭ несмотря на более высокую плотность специальных границ, и толщина их ламелей уменьшается по сравнению с комнатной температурой (Рис. 2е, Табл. 1). Эти наблюдения коррелируют с данными об ориентационной зависимости механического двойникования и температурной зависимости морфологии двойников, описанными в работах $[21,22]$. Понижение температуры деформации усиливает вклад этого механизма в формирование микроструктуры, двойни- ковые границы встречаются чаще и их плотность не изменяется от зерна к зерну (Рис. 2 е, Табл. 1). По данным ПЭМ-анализа после прокатки в зернах присутствует высокая скалярная плотность дислокаций $\rho \sim 10^{14} \mathrm{M}^{-2}$, а охлаждение образцов до температуры жидкого азота вызывает рост значений $\rho$ (Табл. 1), что может быть вызвано формированием высокой плотности границ $\Sigma 3$. Независимо от температуры деформирования, скольжение является основным механизмом деформации стали, а двойникование выступает как дополнительный механизм упрочнения. В полном соответствии с изменением параметров микроструктуры при деформации изменяются механические свойства исследуемых образцов, прокатка вызывает рост предела текучести и способствует снижению пластичности: $\sigma_{0.2}=800 \pm 19$ МПа, $\delta=15 \%$ после прокатки без охлаждения образцов, $\sigma_{0.2}=920 \pm 18$ МПа, $\delta=18 \%$ после низкотемпературной прокатки.

Электролитическое насыщение образцов водородом вызывает его неоднородное распределение в объеме образцов - высокая концентрация характерна для поверхностных слоев, а затем она снижается по мере продвижения к центру [23]. Последующая пластическая деформация способствует транспорту водорода в объем материала за счет диффузии в кристаллической решетке аустенита, стимулированной высокими приложенными давлениями при прокатке, и за счет транспорта водорода на дефектах кристаллического строения

Табл. 1. Характеристики микроструктуры ( $\rho-$ плотность дислокаций, $t-$ толщина двойников, $e-$ расстояние между двойниками, $\rho_{\text {дв }}$ - линейная плотность двойниковых границ) и механические свойства $\left(\sigma_{0.2}-\right.$ предел текучести, $\delta-$ удлинение $)$ образцов стали в зависимости от режима обработки. Данные получены при анализе ПЭМ-изображений и ДОЭ-карт (только $\rho_{\text {дв }}$ ).

Table 1. Characteristics of the microstructure ( $\rho$ - dislocation density, $t-$ twin thickness, $e-$ distance between twins, $\rho_{t \omega}-$ linear density of twin boundaries) and mechanical properties $\left(\sigma_{0.2}-\right.$ yield strength, $\delta$ - elongation $)$ of the steel in dependence on treatment regime. Data were obtained using TEM images and EBSD maps $\left(\rho_{t \omega}\right.$ only).

\begin{tabular}{|c|c|c|c|c|c|c|c|}
\hline $\begin{array}{c}\text { Режим насыщения водородом } \\
\text { Hydrogen-charging regime }\end{array}$ & $\begin{array}{l}\rho, \mathrm{M}^{-2} \\
\rho, \mathrm{m}^{-2}\end{array}$ & $\begin{array}{l}t, \mathrm{HM} \\
t, \mathrm{~nm}\end{array}$ & $\begin{array}{l}e, \mathrm{HM} \\
e, \mathrm{~nm}\end{array}$ & $\begin{array}{l}\rho_{\text {дв }}, M^{-1}(\text { ПЭМ) } \\
\rho_{t \omega}, \mathrm{m}^{-1}(\text { ТЕМ }) \\
\end{array}$ & $\begin{array}{c}\rho_{\text {дв }}, M^{-1}(\text { ДОЭ) } \\
\rho_{t \omega}, \mathrm{m}^{-1}(\mathrm{EBSD}) \\
\end{array}$ & $\begin{array}{l}\sigma_{0.2}, \mathrm{M \Pi а} \\
\sigma_{0.2}, \mathrm{MPa}\end{array}$ & $\delta, \%$ \\
\hline \multicolumn{8}{|c|}{ Режим I / Regime I } \\
\hline $\begin{array}{c}\text { Без наводороживания } \\
\text { Hydrogen-free }\end{array}$ & $0.4 \times 10^{15}$ & $\begin{array}{l}50-100 \\
\left(15-25^{\star}\right)\end{array}$ & $\begin{array}{l}60-100 \\
\left(15-40^{\star}\right)\end{array}$ & $\begin{array}{c}2 \times 10^{6} \\
\left(12 \times 10^{6 *}\right)\end{array}$ & $5.5 \times 10^{6}$ & $800 \pm 19$ & 15 \\
\hline $\begin{array}{l}j=10 \mathrm{MA} / \mathrm{cm}^{2} \\
j=10 \mathrm{~mA} / \mathrm{cm}^{2}\end{array}$ & $0.5 \times 10^{15}$ & $\begin{array}{c}70-200 \\
\left(20-45^{\star}\right)\end{array}$ & $\begin{array}{l}60-250 \\
\left(30-50^{\star}\right)\end{array}$ & $\begin{array}{c}5 \times 10^{6} \\
\left(21 \times 10^{6 *}\right)\end{array}$ & $3.4 \times 10^{6}$ & $880 \pm 19$ & 19 \\
\hline $\begin{array}{l}j=100 \mathrm{MA} / \mathrm{cm}^{2} \\
j=100 \mathrm{~mA} / \mathrm{cm}^{2}\end{array}$ & $0.6 \times 10^{15}$ & $\begin{array}{c}30-180 \\
\left(15-30^{*}\right)\end{array}$ & $\begin{array}{c}70-180 \\
\left(30-50^{*}\right)\end{array}$ & $\begin{array}{c}8 \times 10^{6} \\
\left(25 \times 10^{6 *}\right)\end{array}$ & $0.9 \times 10^{6}$ & $850 \pm 19$ & 19 \\
\hline $\begin{array}{l}j=200 \mathrm{MA} / \mathrm{cm}^{2} \\
j=200 \mathrm{~mA} / \mathrm{cm}^{2}\end{array}$ & $0.8 \times 10^{15}$ & $\begin{array}{c}50-200 \\
(20-50)^{*}\end{array}$ & $\begin{array}{c}70-250 \\
\left(25-50^{\star}\right)\end{array}$ & $\begin{array}{c}8 \times 10^{6} \\
\left(29 \times 10^{6 *}\right) \\
\end{array}$ & $0.6 \times 10^{6}$ & $850 \pm 17$ & 16 \\
\hline \multicolumn{8}{|c|}{ Режим II / Regime II } \\
\hline $\begin{array}{c}\text { Без наводороживания } \\
\text { Hydrogen-free }\end{array}$ & $0.7 \times 10^{15}$ & $20-100$ & $50-150$ & $7 \times 10^{6}$ & $2.1 \times 10^{6}$ & $920 \pm 18$ & 18 \\
\hline $\begin{array}{l}j=10 \mathrm{MA} / \mathrm{cm}^{2} \\
j=10 \mathrm{~mA} / \mathrm{cm}^{2}\end{array}$ & $0.7 \times 10^{15}$ & $10-50$ & $20-80$ & $23 \times 10^{6}$ & $0.3 \times 10^{6}$ & $960 \pm 16$ & 14 \\
\hline $\begin{array}{l}j=100 \mathrm{MA} / \mathrm{cm}^{2} \\
j=100 \mathrm{~mA} / \mathrm{cm}^{2}\end{array}$ & $0.8 \times 10^{15}$ & $10-60$ & $50-110$ & $26 \times 10^{6}$ & $0.6 \times 10^{6}$ & $960 \pm 16$ & 13 \\
\hline $\begin{array}{l}j=200 \mathrm{MA} / \mathrm{cm}^{2} \\
j=200 \mathrm{~mA} / \mathrm{cm}^{2}\end{array}$ & $0.8 \times 10^{15}$ & $10-60$ & $40-150$ & $29 \times 10^{6}$ & $1.5 \times 10^{6}$ & $960 \pm 17$ & 11 \\
\hline
\end{tabular}

* в отдельных зернах, наиболее благоприятно ориентированных для двойникования

${ }^{\star}$ For individual grains favorably oriented for twinning 
(дислокациях). Анализ микроструктуры образцов по глубине не обнаруживает различий в микроструктуре образцов у поверхности и в объеме, то есть прокатка обеспечивает транспорт атомов водорода на все сечение образцов. Непосредственно после насыщения концентрация водорода в образцах слабо зависела от плотности тока при обработке: 0.0017 масс.\% при $j=10 \mathrm{мA} / \mathrm{cm}^{2}, 0.0017$ масс.\% при $j=100 \mathrm{~mA} / \mathrm{cm}^{2}$, 0.0018 масс.\% при $j=200 \mathrm{~mA} / \mathrm{cm}^{2}$. Такое поведение может быть связано с активацией «скин-эффекта» [23] при насыщении образцов с большой плотностью тока. При избыточной концентрации атомарного водорода в электролите, которая реализуется при таком «жестком» режиме насыщения, происходит быстрый рост концентрации водорода в поверхностном слое образца и «экранирование» градиента концентрации между образцом и электролитом, что препятствует проникновению водорода внутрь образца. Несмотря на близкие концентрации адсорбированного водорода в образцах, насыщенных при разных плотностях тока, отличия в их микроструктуре после прокатки существенны (Рис. 1,2).

Насыщение образцов водородом перед прокаткой (по режимам I и II) способствует более активному развитию механического двойникования при последующем деформировании. С увеличением плотности тока при насыщении, в структуре деформированных образцов возрастает плотность двойниковых границ и плотность дислокаций (Рис. 1,2, Табл. 1). Кроме этого, после насыщения при $j=200 \mathrm{MA} / \mathrm{cm}^{2}$ и прокатки при комнатной температуре в структуре стали наблюдали формирование деформационного в-мартенсита (Рис. 1h). А при низкотемпературном деформировании наводороженных образцов, $\gamma \rightarrow \varepsilon$ фазовое превращение наблюдали независимо от плотности тока при обработке (Рис. $2 \mathrm{f}, \mathrm{g}, \mathrm{h}$ ). На ДОЭ-картах по-прежнему наблюдали формирование непрерывных малоугловых разориентировок в пределах исходных аустенитных зерен, но двойниковые пакеты идентифицировали реже, чем после прокатки без насыщения водородом (Рис. $1 \mathrm{a}-\mathrm{d}, 2 \mathrm{a}-\mathrm{d}$ ). Это коррелирует с данными ПЭМ-исследований микроструктуры - тонкие двойниковые ламели однородно распределены в микроструктуре. Таким образом, скольжение и механическое двойникование - основные механизмы деформации стальных образцов, насыщенных водородом, при прокатке. При этом наводороживание стимулирует развитие двойникования и деформационного $\gamma \rightarrow \varepsilon$ превращения, накопление дислокаций скольжения. В результате вызванного наводороживанием изменения микроструктуры стали 01X17Н13M3 ее механические свойства возрастают. Величина условного предела текучести достигает значений $\sigma_{0.2}=850-880 \mathrm{MПа}(\delta=16-19 \%)$ после прокатки без охлаждения образцов и $\sigma_{0.2}=960 \mathrm{MПа}$ $(\delta=11-14 \%)$ после низкотемпературной прокатки.

Экспериментально установленные закономерности по влиянию насыщения водородом на активность механического двойникования и $\gamma \rightarrow \varepsilon$ превращения коррелирует с данными о водородно-индуцируемом понижении величины ЭДУ сталей, отмечаемым ранее в работах $[14,15]$. Формирование высокой плотности двойниковых границ приводит к росту плотности дислокаций из-за их блокировки у $\Sigma 3$-границ [24]. При этом наблюдаемые различия плотности специальных границ в образцах, насыщенных при разной плотности тока, свидетельствуют о том, что несмотря на близкие значения общей концентрации адсорбированного образцом водорода при разных режимах насыщения, его распределение в образцах различно. В образцах, насыщенных при $j=200$ мA/см², градиент концентрации атомов водорода в приповерхностном слое может быть больше, чем в образцах, насыщенных при $j=10 \mathrm{MA} / \mathrm{cm}^{2}$, и механизм деформации стали при прокатке может определяться не только содержанием водорода в структуре стали, но и величиной градиента его концентраций и связанных с ним искажений кристаллической решетки, микронапряжений и т.п. Это вопрос требует отдельного исследования.

\section{4. Заключение}

Холодная деформация аустенитной стали 01Х17Н13M3 приводит к формированию структуры с высокой плотностью дефектов кристаллического строения. Основным механизмом деформации при прокатке выступает дислокационное скольжение, оно сопровождается развитием механического двойникования как дополнительного механизма, способствующего фрагментации структуры. Насыщение водородом и понижение температуры деформации способствуют более активному развитию механического двойникования и протеканию $\gamma \rightarrow \varepsilon$ фазового превращения в стали. А увеличение плотности тока при насыщении образцов водородом перед прокаткой приводит к росту плотности двойниковых границ и дислокаций. При этом увеличение плотности тока при наводороживании от $10 \mathrm{~mA} / \mathrm{cm}^{2}$ до $200 \mathrm{~mA} / \mathrm{cm}^{2}$ не способствует значительному повышению концентрации адсорбированного материалом водорода (0.0017-0.0018 масс.\%), что в совокупности с микроструктурными исследованиями свидетельствует о различном распределении водорода в образцах непосредственно перед деформированием и о важной роли градиента концентрации водорода у поверхности на механизмы деформации стали при последующей прокатке. Полученные данные свидетельствуют о том, что при близких значениях концентрации адсорбированного водорода в структуре сталей, режим насыщения влияет на механизм деформации и деформационные фазовые превращения в аустенитной стали.

Благодарности/Acknowledgements. Работа выполнена в рамках государственного задания ИФПМ СО РАН, тема номер FWRW-2019-0030. Исследования проведень на оборудовании ИФПМ СО РАН (ЦКП «Нанотех») и НИУ «БелГУ» (ЦКП «Диагностика структурь и свойств наноматериалов»). ( The work was carried out within the framework of the state assignment of the ISPMS $S B$ RAS, topic number FWRW-2019-0030. The studies were carried out on the equipment of the ISPMS SB RAS (Nanotech Center for Collective Use) and BelGU (Center for Collective Use "Diagnostics of the Structure and Properties of Nanomaterials"). 


\section{Литература/References}

1. B.N. Arzamasov, T.V. Soloviev, S.A. Gerasimov et al. Reference book on constructional materials. Moscow, MGTU im. N.E. Bauman (2006) 640 p. (in Russian) [Б. Н. Арзамасов, Т.В. Соловьев, С.А. Герасимов и др. Справочник по конструкционным материалам. Москва, МГТУ им. Н. Э. Баумана (2006) 640 с.]

2. A.P. Shlyamnev et al. Corrosion-resistant, heat-resistant and high-strength steels and alloys: a reference book. Moscow, Intermet Engineering (2000) 232 p. (in Russian) [А.П. Шлямнев и др. Коррозионностойкие, жаростойкие и высокопрочные стали и сплавы: справочник. Москва, Интермет Инжиниринг (2000) $232 \mathrm{c.}$.]

3. S.L. Chawla, R.K. Gupta. Materials Selection for Corrosion Control. ASM International Materials Park, OH (1997) 508 p.

4. K. H. Lo, C. H. Shek, J. K. L. Lai. Mater. Sci. and Eng.: R. 65 (4-6), 39 (2009). Crossref

5. A. F. Padihla, R.L. Plaut, P. R. Rios. ISIJ International. 43 (2), 135 (2003). Crossref

6. G. Korznikova, S. Mironov, T. Konkova et al. Metall. Mater. Trans. A. 49, 6325 (2018). Crossref

7. M. El-Tahawy, G. Jenő, H. Yi, et al. Mater. Sci. Forum. 885, 13 (2017). Crossref

8. I. Yu. Litovchenko, A.N. Tyumentsev, E. P. Naiden. Phys. Meso. 9 (1), 31 (2014). (in Russian) [И. Ю. Литовченко, A.Н. Тюменцев, Е.П. Найден. Физическая мезомеханика. 9 (1), 137 (2014).] Crossref

9. I. Yu. Litovchenko, N.V. Shevchenko, A.N. Tyumentsev, E. P. Naiden. Phys. Meso.Special.9, 137 (2006). (in Russian) [И. Ю. Литовченко, Н. В. Шевченко, А.Н. Тюменцев, Е.П. Найден. Физическая мезомеханика. Спец. выпуск 9, 137 (2006).] Crossref
10. J. W. Christian, S. Mahajan. Prog. Mater. Sci. 39, 1 (1995). Crossref

11. S. V. Astafurov, G. G. Maier, E. V. Melnikov, V. A. Moskvina, M. Yu. Panchenko, E. G. Astafurova. Mater. Sci. and Eng. A. 756, 365 (2019). Crossref

12. L. Zhang, Zh. Li, J. Zheng, et al. Int. J. of Hydrogen Ener. 38, 8208 (2013). Crossref

13. M. Hatano, M. Fujinami, K. Arai, H. Fujii, M. Nagumo. Acta Mater. 67, 342 (2014). Crossref

14. A.E. Pontini, J.D. Hermida. Scripta Mater. 37 (11), 1831 (1997). Crossref

15. E. G. Astafurova, G. G. Maier, E. V. Melnikov, et al. Phys. Meso. 21, 263 (2018). Crossref

16. L. Zhang, M. Imade, B. An et al. ISIJ International. 52 (2), 240 (2012). $\underline{\text { Crossref }}$

17. S. Sigiyama, H. Ohkubo, M. Takenaka, et al. J. of Nuclear Mater. 283-287, 864 (2000). Crossref

18. Y. Mine, Z. Horita, Y. Murakam. Acta Mater. 57 (10), 2993 (2009). Crossref

19. E. Melnikov, G. Maier, V. Moskvina, E. Astafurova. AIP Conf. Proc. 1909, 020136 (2017). Crossref

20. L.M. Utevsky. Diffraction electron microscopy in metal science. Moscow, Metallurgiya (1973) 584 p. (in Russian) [Л.M. Утевский. Дифракционная электронная микроскопия в металловедении. Москва, Металлургия (1973) 584 с.]

21. M. Koyama, E. Akiyama, T. Sawaguchi, et. al. Corrosion Sci. 75, 345 (2013). Crossref

22. E. G. Astafurova, I. V. Kireeva, Yu. I. Chumlyakov, H.J. Maier, H. Sehitoglu. Int. J. Mat. Res. 98 (2), 144 (2007). Crossref

23. V.A. Polyanskiy, A.K. Belyaev, EL. Alekseeva, A.M. Polyanskiy, D. A. Tretyakov, Yu. A. Yakovlev. Contin. Mechan. Thermodyn. 31, 1961 (2019). Crossref

24. Z. W. Wang, Y. B. Wang, X.Z. Liao, Y. H. Zhao. Scr. Mater. 60 (1), 52 (2009). $\underline{\text { Crossref }}$ 